\title{
New Frontiers in Pediatric Dentistry
}

Pediatric dentists play the most responsible role of introducing dentistry to an individual for the very first time. Starting with parents during the prenatal period and after the child is born, the duties extend to understanding the child, his/her genetics and environment, modifying habits and behavior and successfully instilling healthy oral hygiene practices. It is an ever so rewarding experience of witnessing them attain these milestones and develop to have positive dental attitudes.

Over the years, pedodontists have been able to look into the future and have become skilled to 'catch them young'. We have come a long way in understanding caries process and the many measures to prevent it. We now have access to newer and more biocompatible restorative materials that enable us to widely practice minimally invasive dentistry. Rotary endodontics have improved the ease of performing pulpectomies for primary dentition and treating young permanent teeth. Preformed zirconia crowns have changed esthetic pediatric practice and has become a common everyday procedure. Lately, better awareness has been created among the parents to have their children examined by pediatric dentists and to follow the various preventive protocols. The future of pediatric dentistry as a specialty is bright.

Artificial intelligence is gaining momentum in all fields and is opening up new frontiers in pediatric dentistry as well. Beginning with examination and diagnosis, behavior management, pain control, orthodontic tooth movement and restorative dentistry, artificial intelligence has a myriad of potential applications and it can change the way we teach and practice modern dentistry. Virtual reality as an aid to teaching in dental schools and as a behavior management tool is the most engaging and effectual. Technological advances can be integrated by pediatric dentists to improve the skills of special children like touchscreen tablets, trackballs and intellikeys USB for easier two-way communication and video modeling and google sketch up for behavior management. There are many useful innovations taking place that will be available to us in the near future with which we can empower our patients and parents.

This year we are celebrating 100 years of dentistry in India. However, in our country dental caries is still a major problem with sugars and lack of oral hygiene being the mighty evil. There is a need to popularize the use of professionally assisted preventive measures like silver diamine fluoride, nano silver fluoride, newer generation sealants, demineralizing agents and pastes which have proved effective for the masses. We also need to tackle the disparities in children's oral health and access to dental care in rural India. As a part of Government of India's plan of attaining oral health for all by 2022, relentless prevention campaigns are being organized encompassing all state schools to mark a new dawn in achieving improved dental health for all.

As responsible practitioners we need to absorb all the latest innovations, evaluate and appraise them to bring more smiles and build a healthier generation.

Nagaveni NB

Professor

Department of Pedodontics and Preventive Dentistry

College of Dental Sciences

Davangere, Karnataka, India 